\title{
Development and Preclinical Evaluation of Virus Like Particle Vaccine Against COVID-19 Infection
}

\author{
Ismail Yilmaz C ${ }^{1}$, Emre Ipekoglu ${ }^{1}$, Artun Bulbul ${ }^{2}$, Nilsu Turay ${ }^{2}$, Muzaffer Yildirim $^{2}$, Irem \\ Evcili $^{2}$, Naz Yilmaz ${ }^{1}$, Yagmur Aydin ${ }^{1}$, Nese Guvencli ${ }^{1}$, Bilgi Gungor ${ }^{2}$, Berfu Saraydar ${ }^{2}$, Asli \\ Gulce Bartan ${ }^{2}$, Bilgehan Ibibik ${ }^{2}$, Tugce Bildik ${ }^{2}$, Illayda Baydemir ${ }^{1}$, Hatice Asena Sanli ${ }^{3}$, \\ Basak Kayaoglu ${ }^{3}$, Yasemin Ceylan ${ }^{3}$, Tugce Yildirim ${ }^{3}$, Irem Abras ${ }^{3}$, Ihsan Cihan Ayanoglu ${ }^{3}$, \\ Sefa Burak Cam ${ }^{4}$, Eda Ciftci Dede ${ }^{4}$, Merve Gizer ${ }^{4}$, Osman Erganis ${ }^{5}$, Fahriye Sarac ${ }^{6}$, Serdar \\ Uzar $^{6}$, Hakan Enul ${ }^{6}$, Cumhur Adiay ${ }^{6}$, Gamze Aykut ${ }^{2}$, Hivda Polat ${ }^{7}$, Ismail Selim Yildirim ${ }^{3}$, \\ Saban Tekin ${ }^{7}$, Hasan E. Zeytin ${ }^{8}$, Petek Korkusuz ${ }^{4}$, Ihsan Gursel ${ }^{2}$, and Mayda Gursel ${ }^{1}$ \\ ${ }^{1}$ Middle East Technical University Department of Biological Sciences Ankara Turkey \\ ${ }^{2}$ Bilkent University Molecular Biology and Genetics Department Ankara Turkey \\ ${ }^{3}$ Affiliation not available \\ ${ }^{4}$ Hacettepe University Histology and Bioengineering Departments Ankara Turkey \\ ${ }^{5}$ Selcuk University Faculty of Veterinary Sciences Konya Turkey \\ ${ }^{6}$ Istanbul Pendik Veteriner Kontrol Enstitusu \\ ${ }^{7}$ Tubitak Marmara Arastirma Merkezi \\ ${ }^{8}$ Nobel Ilac San ve Tic AS
}

July 14, 2021

\begin{abstract}
Background Vaccines that incorporate multiple SARS-CoV-2 antigens can further broaden the breadth of virus-specific cellular and humoral immunity. This study describes the development and immunogenicity of SARS-CoV-2 VLP vaccine that incorporates the 4 structural proteins of SARS-CoV-2. Methods VLPs were generated in transiently transfected HEK293 cells, purified by multimodal chromatography and characterized by tunable resistive pulse sensing, AFM, SEM, and TEM. Immunoblotting studies verified the protein identities of VLPs. Cellular and humoral immune responses of immunized animals demonstrated the immune potency of the formulated VLP vaccine. Results Transiently transfected HEK293 cells reproducibly generated vesicular VLPs that were similar in size to and expressing all four structural proteins of SARS-CoV-2. Alum adsorbed, K3-CpG ODN adjuvanted VLPs elicited high titer anti-S, anti-RBD, anti-N IgG, triggered multifunctional Th1 biased $\mathrm{T}$ cell responses, reduced virus load and prevented lung pathology upon live virus challenge in vaccinated animals. Conclusion These data suggest that VLPs expressing all four structural protein antigens of SARS-CoV-2 are immunogenic and can protect animals from developing COVID-19 infection following vaccination.
\end{abstract}



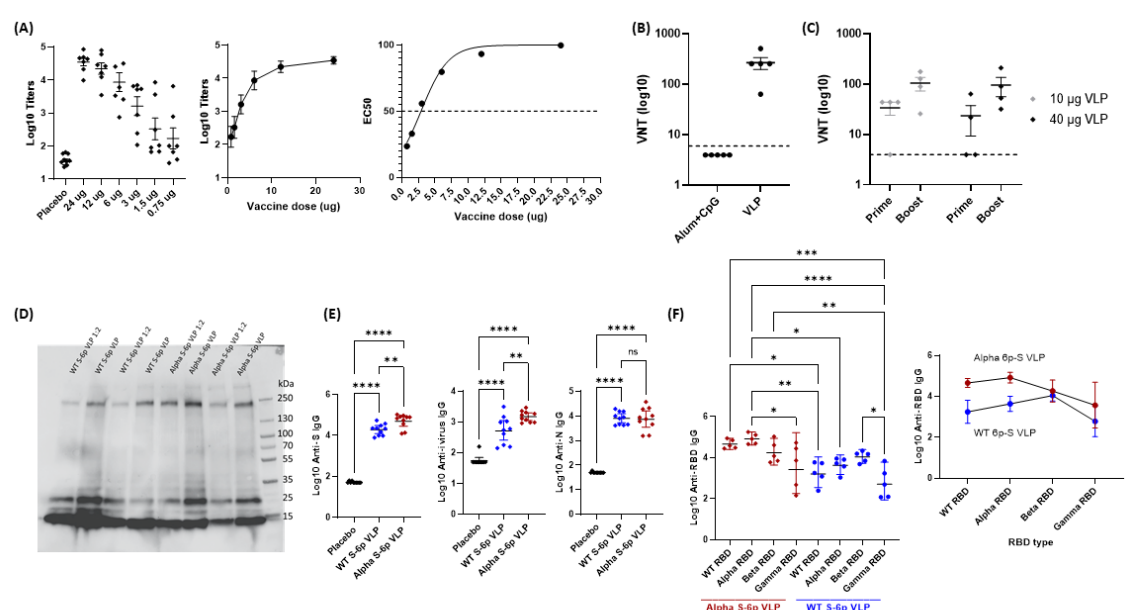

Figure 1: This is a caption

\section{Development and Preclinical Evaluation of Virus Like Particle Vaccine Against COVID-19 Infection}

Ismail C Yilmaz ${ }^{1,2^{*}}$, Emre M Ipekoglu ${ }^{1 *}$, Muzaffer Yildirim $^{2 *}$, Irem Evcili2*, Naz Yilmaz ${ }^{1}$, Artun Bulbul ${ }^{2}$, Nilsu Turay ${ }^{2}$, Yagmur Aydin ${ }^{1}$, Nese Guvencli ${ }^{1}$, Bilgi Gungor ${ }^{2}$, Berfu Saraydar ${ }^{2}$, Asli Gulce Bartan ${ }^{2}$, Bilgehan Ibibik ${ }^{2}$, Tugce Bildik ${ }^{2}$, İlayda Baydemir ${ }^{1}$, Sefa Burak $\mathrm{Cam}^{3}$, Eda Ciftci Dede ${ }^{3}$, Merve Gizer ${ }^{3}$, Osman Erganis $^{4}$, Fahriye Sarac ${ }^{5}$, Serdar Uzar, Hakan Enul, Cumhur Adiay, Gamze Aykut ${ }^{2}$, Hivda Polat ${ }^{6}$, Saban Tekin $^{6}$, Hasan E. Zeytin ${ }^{7}$, Petek Korkusuz ${ }^{3}$, Ihsan Gursel ${ }^{2 \#}$, Mayda Gursel ${ }^{1 \#}$

${ }^{1}$ Middle East Technical University, Department of Biological Sciences, Ankara, Turkey

${ }^{2}$ Bilkent University, Molecular Biology and Genetics Department, Ankara, Turkey

${ }^{3}$ Hacettepe University, Histology and Bioengineering Departments, Ankara, Turkey

${ }^{4}$ Selcuk University, Faculty of Veterinary Sciences, Konya, Turkey

${ }^{5}$ Pendik Veterinary Research and Control Institute, Istanbul, Turkey

${ }^{6}$ TUBITAK, Marmara Research Center, Istanbul, Turkey

${ }^{7}$ Nobel Pharma, Biotechnology Research Center, Istanbul, Turkey

*Ismail C Yilmaz, Emre M Ipekoglu, Muzaffer Yildirim, Irem Evcili should be considered joint first authors.

\# Mayda Gursel and Ihsan Gursel should be considered joint senior authors.

\#Corresponding Authors: M Gursel (mgursel@metu.edu.tr) and I Gursel (ihsangursel@bilkent.edu.tr)

Running Title: Protective VLP vaccine against COVID-19

WORD COUNT: 3551

\section{ABBREVIATIONS:}

2p, 2 proline; 6p, Hexaproline; AFM, Atomic force microscopy; Alum, Aluminium hydroxide; CD33 SP, Cleavable signal peptide; COVID-19, Coronavirus Disease-2019; CpG, Cytosine-phosphate-Guanine; CT, Cytoplasmic tail; CTL, Cytotoxic T lymphocyte; E, Envelope protein; EC50, Half maximal effective concentration; ELISA, Enzyme linked immunosorbent assay; FD, T4 fibritin trimerization domain; FP, Fusion 
peptide; GMT, Geometric mean titer, GT,Gomori trichrome; H\&E, Hematoxylin and eosin; hACE2, Human angiotensin-converting enzyme 2; HD, High dose; HEK293, Human embryonic kidney cell line; His, Histidine tag; HR, Heptad repeat; IFN- $\gamma$, Interferon gamma; IL, Interleukin; LD, Low dose; M, Membrane protein; MFI, Mean fluorescence intensity; N, Nucleocapsid protein; NC, Nucleocapsid; NTD, N- terminus domain; ODN, Oligodeoxynucleotide; PEIpro, polyethylene imine transfection reagent; Pfu, Plaque forming unit; RBD, Receptor binding domain; S, Spike protein; SARS-CoV-2, Severe acute respiratory syndrome coronavirus 2; SEM, Scanning electron microscopy; TEM, Transmission electron microscopy; TEV, Tobacco etch virus; TH1, T helper 1; TH2, T helper 2; THM, Thrombin cleavage site; TM, Transmembrane domain; TRPS, Tunable resistive pulse sensing; VAERS, Vaccine adverse event reporting system; VLP, Virus-like particle; VNT, Virus neutralization titer; WT, Wild type

\section{Acknowledgements:}

This work is supported by TUBITAK, Center of Excellence Support Program (1004 Program) to MG as part of the consortium titled "Development of Drug and Vaccine against COVID-19" (Project No: 18AG020). IG received partial support from Ministry of Development (Grant name: UMRAM-ASI, Project \#: 2015BSV302). The authors would like to thank Ihsan Cihan Ayanoglu, Nobel Pharma Biotechnology Research Center Team heads and all their team members, particularly to Mohan Babu Kasa, Mohit Sharma, Naidu Mookala, Vivek Yadav, Tirumal Rao Yannabathina. We sincerely appreciate the encouragement and support from Prof Hasan Mandal, and Minister Mustafa Varank.

\section{ABSTRACT}

Background Vaccines that incorporate multiple SARS-CoV-2 antigens can further broaden the breadth of virus-specific cellular and humoral immunity. This study describes the development and immunogenicity of SARS-CoV-2 VLP vaccine that incorporates the 4 structural proteins of SARS-CoV-2.

Methods VLPs were generated in transiently transfected HEK293 cells, purified by multimodal chromatography and characterized by tunable resistive pulse sensing, AFM, SEM, and TEM. Immunoblotting studies verified the protein identities of VLPs. Cellular and humoral immune responses of immunized animals demonstrated the immune potency of the formulated VLP vaccine.

Results Transiently transfected HEK293 cells reproducibly generated vesicular VLPs that were similar in size to and expressing all four structural proteins of SARS-CoV-2. Alum adsorbed, K3-CpG ODN adjuvanted VLPs elicited high titer anti-S, anti-RBD, anti-N IgG, triggered multifunctional Th1 biased $\mathrm{T}$ cell responses, reduced virus load and prevented lung pathology upon live virus challenge in vaccinated animals.

Conclusion These data suggest that VLPs expressing all four structural protein antigens of SARS-CoV-2 are immunogenic and can protect animals from developing COVID-19 infection following vaccination.

\section{KEYWORDS:}

CpG ODN Adjuvant, COVID-19, SARS-CoV-2, , Vaccine, Virus like particle,

\section{Introduction}

Rapid development of effective vaccines is indispensable in constraining the COVID-19 pandemic. Multiple highly effective COVID-19 vaccines have recently been approved for human use and several are still in clinical development. ${ }^{1}$ The majority of current SARS-CoV-2 vaccines target only the Spike (S) antigen with the main intent of eliciting neutralizing antibodies against the receptor binding domain (RBD) to neutralize infection. $^{2-6}$ However, the emergence of variants of concern Alpha (B.1.1.7), Beta (B.1.351), Gamma (P.1) and Delta (B.1.617.2) with altered S sequences, raises concerns on dependence on S-based vaccines, particularly in light of recent evidence indicating the potential for variants to at least partially escape from neutralizing antibodies. ${ }^{7-18}$ Although neutralizing and spike binding antibodies strongly correlate with protective immune mechanisms ${ }^{19}$, cellular immunity also likely contributes to virus clearance. ${ }^{20-23}$ In addition to spike, targeting of other SARS-CoV-2 antigens in vaccines, such as the membrane (M) and nucleocapsid (N), could hypothetically present an advantage over S-dependency of vaccines two-fold: First, $\mathrm{M}$ and $\mathrm{N}$ harbor 
immunodominant CD4+ and CD8+ T-cell epitopes that can further broaden the breadth of cellular and humoral immunity $;{ }^{24}$ Second, non-neutralizing anti-N antibodies can potentially contribute to heterosubtypic immunity, as previously demonstrated for other enveloped viruses. ${ }^{25-26}$ To this end, herein, we describe the preclinical development of a virus like particle (VLP) vaccine expressing the Hexaproline prefusion stabilized spike (S-6p) ${ }^{27}$ in addition to N, M and envelope (E) of SARS-CoV-2 structural proteins. To improve immunogenicity, S-6p VLPs were adsorbed to alhydrogel (alum) and formulated with a K-type CpG ODN (also referred to as B type) as a vaccine adjuvant to boost both humoral and cellular (Th1 cells and CTL) immunity. ${ }^{28-30}$

\section{MATERIALS AND METHODS}

\section{Cloning of VLP encoding genes}

Human codon optimized genes coding for WT, 2p-S, HexaPro Spike (6p-S)27, membrane glycoprotein (M) (NCBI Refseq: YP_009724393.1), envelope (E) (NCBI Refseq: YP_009724392.1) and nucleocapsid (N) (NCBI Refseq: YP_009724397.2) proteins of SARS-CoV-2 were synthesized by Integrated DNA Technologies, Inc. (California, USA) with a C- terminus histidine tag. In order to achieve mammalian expression of S, M, E and $\mathrm{N}$ genes, pVitro1 and pVitro2 mammalian dual expression plasmids (Invivogen, France) were used. The sequences for S (WT, 2p-S, 6p-S) were cloned at the BamHI site and the sequence of $\mathrm{E}$ was cloned at the BglII site of the pVitro2 plasmid. The sequence for $\mathrm{M}$ was inserted at the BamHI while $\mathrm{N}$ was cloned at the BglII site of pVitro1. Plasmid sequences were verified by next generation sequencing (NGS) (Intergen, Ankara).

\section{Transient transfection of suspension HEK293 cells}

HEK293 suspension adapted cells (Florabio, Turkey) were grown in serum free Orchid293 CD transfection medium (Florabio, İzmir) supplemented with $400 \mathrm{mg} / \mathrm{L}$ L-glutamine (Sigma, Canada). Cells were transiently transfected with $1 \mu \mathrm{g}$ each of pVitro1 + pVitro2 and PEIpro (Polyplus, France) at a ratio of 2:1 (v/w). 96120 hours of post-transfection, VLP containing culture supernatants were harvested and filtered through a $0.22 \mu \mathrm{m}$ filter membrane.

\section{Purification of VLPs}

To eliminate host cell-derived nucleic acids, the harvest was treated with $200 \mathrm{U} / \mathrm{ml}$ of Denarase (c-LEcta, Germany) for 2 hours at $37^{\circ} \mathrm{C}$. VLPs were purified on a Hi-Screen Capto Core 400 (Cytiva, USA) column using ÄKTA-GO fast protein liquid chromatography system (Cytiva, USA). Flow-through fractions containing VLPs were pooled and subjected to ultrafiltration/dialfiltration on a Sartocon@ Slice 200 Hydrosart $\mathbb{R}$ 100kDA (Sartorius, Germany) cassette.

\section{SDS-PAGE, Western blotting and VLP quantification}

For SDS-PAGE, the samples were mixed with reducing $4 \mathrm{X}$ Laemmli Buffer and denatured at $95^{\circ} \mathrm{C}$ for 5 minutes. $18 \mu \mathrm{l}$ of sample was loaded into each well of 4-20\% Mini-PROTEAN TGX Stain-Free Protein Gel (Bio-Rad, USA). Following completion of SDS-PAGE, gels were transferred to a PVDF $0.2 \mu \mathrm{m}$ membrane using the Mini Trans-Blot ( ) Cell System (Bio-Rad, USA) for an hour at 100V. As primary antibodies, HRP-conjugated 6xHis, His-Tag antibody (Proteintech, USA), Spike-S1 and Nucleocapsid antibody (ProSci, USA) were used. Anti-rabbit and anti-mouse secondary antibodies were used for anti-spike S1 and anti-N immunoblots. The HRP activity was detected with ECL Prime HRP Reagent (Cytiva, U.S.A.) and imaged by an Amersham Imager 600 (Cytiva, USA). VLP content was quantified with the Pierce ${ }^{\mathrm{TM}}$ micro BCA protein assay kit (Thermo Fisher Scientific, USA) according to manufacturer's instructions.

\section{Characterization of VLPs by SEM, AFM and TEM}

A $10 \mu \mathrm{l}$ of the VLP solution was deposited onto a silica surface, air-dried and sputter-coated with $8 \mathrm{~nm}$ of $\mathrm{Au} / \mathrm{Pd}$ alloy using a precision coating system prior to imaging on an environmental SEM (Technia; FEI, USA). 
Purified VLPs were diluted 1:100 in PBS and adsorbed onto mica sheets. The adsorbed samples were airdried and micrometer-scale AFM imaging was conducted in non-contact dynamic mode (NanoMagnetics Instruments, Turkey) according to manufacturer's instructions. Scans were analyzed using the NMI Image Analyzer software.

VLP producing HEK293 cells were processed for standard TEM. Briefly, cells were fixed in $2 \%$ glutaraldehyde $30 \mathrm{~min}$ at RT, fixed in 1\% osmium tetroxide, dehydrated through a graded series of ethanol (30-100\%) and embedded in Epon 812 resin. Sections were stained with uranyl acetate and lead citrate. Imaging was performed at $80 \mathrm{kV}$ using a JEOL-JEM 1400 transmission electron microscope. Digital images of the specimens were acquired using a CCD camera (Gatan Inc., USA).

\section{Nanoparticle Analysis of VLPs Using TRPS}

Tunable resistive pulse sensing (TRPS) measurements were executed with the qNano Gold (IZON S/N 601A) system and analyzed with the IZON Control Suite 3.4.2.48 software (IZON Science LTD). The calibration particles, IZON coating solution, wetting solution and nanopores were used according to the manufacturer's protocols. 35 $\mathrm{\mu L}$ of 1:1000 diluted calibration particles (CPC100, IZON Reagent Kit, RK3-167) or sample liquid was loaded and each sample reading was repeated three times.

\section{Bead Based Binding Assays}

Carboxyl modified latex beads (2 mg of $4 \%$ (w/v), Thermo Fisher Scientific, USA) were coated with $5 \mu \mathrm{g}$ recombinant hACE2 (ProSci, USA) or recIL-1 $\beta$ in PBS and blocked in 5\% BSA in PBS. Beads were washed once and resuspended in $5 \%$ BSA/PBS/0.05\% NaN3 (FACS buffer). VLPs were loaded with $50 \mu \mathrm{M}$ carboxyfluorescein succinimidyl ester (CFSE) for 30 mins at $37^{\circ} \mathrm{C}$ and free dye was removed using a HiTrap $\mathbb{R}$. Desalting column (Cytiva, U.S.A.). Recombinant hACE2 and anti-IL1 $\beta$ coated beads were diluted 1:50. CFSE labelled VLPs were serially diluted five times, each dilution was mixed with an equal volume of coated bead followed by overnight incubation at $4^{\circ} \mathrm{C}$. Beads were washed three times and analyzed on a Novocyte 3000 flow cytometer.

\section{Immunization studies}

All animal studies were conducted with prior approval of the animal ethics committee of Bilkent University (BIAEC, 2020-7/14.42020).

VLPs were adsorbed onto $2 \%$ Alhydrogel $(\mathrm{R})(10.2 \mathrm{mg} / \mathrm{mL}$, Alum hereafter) and adjuvanted with K3-CpG ODN $(2.6 \mu \mathrm{g} / \mathrm{mL})$. Groups of female mice (BALB/c, C57BL/6, or K18 hACE2 Tg, 6-8 weeks old, N=512 /group in separate experiments) were subcutaneously (s.c.) injected with $200 \mu \mathrm{l}$ VLP vaccine (0.75-24 $\mu \mathrm{g}$ VLP antigen) two weeks apart. On indicated days, mice were bled and sera were stored at $-20^{\circ} \mathrm{C}$ until further use. In some experiments, WT, 2p- or 6p-spike expressing VLPs were formulated only with Alum, or K3-CpG ODN. In rat and ferret immunization experiments, 10 or $40 \mu \mathrm{g}$ VLP adsorbed onto $600 \mu \mathrm{g}$ Alum and adjuvanted with $300 \mu \mathrm{g}$ K3-CpG ODN) was s.c. injected two weeks apart and blood was collected on indicates days.

\section{IgG ELISA}

Maxibinding semi-hydrophobic ELISA plates (SPL Life Sciences, Korea) were coated with $50 \mu \mathrm{l} /$ well in-house recombinant $6 \mathrm{p}-\mathrm{S}(5 \mu \mathrm{g} / \mathrm{ml})$ and nucleocapsid $(20 \mu \mathrm{g} / \mathrm{ml})$, RBD $(3 \mu \mathrm{g} / \mathrm{ml})$ and inactive SARS-CoV-2 virus $(5 \mu \mathrm{g} / \mathrm{ml})$ in PBS at $4^{\circ} \mathrm{C}$ overnight. 1:50 diluted mice sera were 5 -fold serially diluted in $5 \%$ BSA in PBSTween (0.05\%). ALP conjugated anti-mouse IgG, anti-mouse IgG1 and anti-mouse IgG2a (Southern Biotech, USA) antibodies were utilized at a 1:1000 dilution. PNPP substrate was added according to manufacturer's instructions. OD values were measured at $405 \mathrm{~nm}$ with a microplate reader (Molecular Devices, USA).

\section{Cytometric Bead Array for Measurement of CD4+ Helper T Responses}

CD4+ T helper cell cytokine levels were assessed with the LEGENDplex MU Th Cytokine Panel (12-plex) w/ VbP V03 kit (Biolegend, U.S.A.) according to the manufacturer's instructions from the supernatants of 
splenocytes stimulated with nucleocapsid $(20 \mu \mathrm{g} / \mathrm{ml})$ and 6p-Spike proteins $(5 \mu \mathrm{g} / \mathrm{ml})$.

\section{SARS-CoV-2 challenge in K18 hACE2 transgenic mice}

ACE2 Tg BALB/c mice (6-8wks old, 10mice/group) were immunized twice subcutaneously 14 days apart with low (10ug) or high (40ug) dose of VLP+Alum+K3-CpG vaccine or with placebo. On day 36, mice were challenged for three consecutive days with live SARS-CoV-2 virus $\left(10^{5} \mathrm{pfu} / 50 \mathrm{ul}\right)$ intranasally. One week after last virus instillation, animals were sacrificed and major organs were recovered. Viral loads in lung specimens were assessed by qRT-PCR using nucleocapsid primers NC1and NC2.

\section{Histomorphometric Evaluation}

Lung samples were fixed in buffered formaldehyde solution. Tissues were dehydrated in an automated tissue processor (TP1020, Leica, Germany). Sections were obtained in a temperature-controlled paraffin station (LG1150H-C, Leica, Germany) on a sliding microtome (SM2000R, Leica, Germany), were deparaffinized at 600C overnight and stained with Hematoxylin-Eosin and Gomori's Trichrome techniques. All sections were evaluated using a bright field microscope with a camera attachment using an image analysis program (DM6B, DFC7000T, LAS X, Leica, Germany). Inflammation was semi-quantitatively scored between 0 and 5 in the perivascular, peribronchiolar, subpleural regions and in the whole section. American Thoracic Society's acute lung injury scoring was followed to report total lung injury. ${ }^{43}$ The parenchymal inflammation area was evaluated for each animal by combining the images obtained at $4 \mathrm{x}$ magnification using Tile Scanning feature of the analysis program and the area of inflammation was calculated quantitatively in $\mu \mathrm{m}^{2}$ and then proportioned to the total lung area.

\section{Virus Neutralization Assay}

Heat-inactivated two-fold serially diluted sera were mixed with an equal volume of 100 TCID50 of SARSCoV-2 for 1 hour at $37^{\circ} \mathrm{C} .100 \mu \mathrm{L}$ of each dilution was transferred in quadruplicate onto VERO E6 cells. After 4 days of incubation, plates were inspected by an inverted optical microscope and the highest serum dilution that protected more than $90 \%$ of cells from cytopathic effect CPE was taken as the neutralization titre.

\section{Statistical Analysis}

Statistical differences of all treatment groups were analyzed using Graph Pad Prism 9 statistical software. Groups were compared by one-way ANOVA with Dunnett's multiple comparisons test. In all analyses a p value below 0.05 was considered to be statistically significant.

\section{Results}

\section{Expression, purification and characterization of SARS-CoV-2 VLPs}

A panel of spike (S) protein encoding genes (with unmodified and modified versions) of SARS-CoV-2 S, membrane glycoprotein $(\mathrm{M})$, envelope $(\mathrm{E})$ and nucleocapsid $(\mathrm{N})$ were engineered (Figure 1A). Spike genes were designed and synthesized in three forms: WT with unmodified S, $2 \mathrm{P}$ with two proline substitutions $(\mathrm{K} 986 \mathrm{P}, \mathrm{V} 987 \mathrm{P})^{31-34}$, or 6P with six proline substitutions (F817P, A892P, A899P, A942P, K986P, V987P) ${ }^{27}$ to stabilize the prefusion conformation. Modifications made to the polybasic furin cleavage site between S1 and S2, locations of the CD33 signal sequence, T4 fibritin (foldon) trimerization motif and the histidine tag sequence are also indicated in Figure 1A. For the nucleocapsid construct, amino acid sequence of the authentic virus was used without modification. S, M, E and $\mathrm{N}$ genes were codon optimized for mammalian cells and cloned into pVitro1 ( $\mathrm{N}$ and $\mathrm{M}$ ) and pVitro2 (WT, 2P or 6P S, and E) dual mammalian expression plasmids. Transient transfection of pVitro1 and pVitro2 S,M,E and N-encoding plasmids into HEK293 cells resulted in cellular assembly, secretion and subsequent accumulation of VLPs in culture supernatant. For the purification of SARS-CoV-2 VLPs (Figure 1B), Denarase treated and clarified cell culture supernatant was loaded onto a HiScreen CaptoCore 400 multimodal size-exclusion/ion-exchange chromatography column (Cytiva, Sweden) and pooled flow-through fraction was subjected to ultrafiltration/diafiltration. VLPs were characterized by transmission electron microscopy (TEM; Figure 1C), scanning electron microscopy (SEM; Figure 1D), atomic 
force microscopy (AFM; Figure 1E), tunable resistive pulse sensing (TRPS; Figure 1F) and immunoblotting for SARS-CoV-2 antigen content using anti-His-Tag, anti-N (Figure 1G) and anti-S1 antibodies (Figure 1H). HEK293 producer cells released intact VLPs into the culture supernatant (Figure 1C). Purified VLPs were spherical, vesicular structures (Figures 1D and E) that were similar in size to SARS-CoV-2 virions (117 \pm 38 , $127 \pm 41$ and $119 \pm 36$ for the WT, $2 p$ or $6 p$ incorporating VLPs, respectively; Figure $1 \mathrm{~F}$ ). Spike protein expression in 2p-VLPs was enhanced relative to WT spike displaying VLPs, whereas 6p-S base construct enabled maximal spike incorporation (Figure 1G). Membrane, envelope and nucleocapsid expressions were relatively stable and did not change substantially among WT, 2p or 6p spike expressing VLPs (Figure 1G). Comparison of spike-specific immunoblots of $6 \mathrm{p}-\mathrm{VLPs}$ with inactivated SARS-CoV-2 virions revealed that 6p-VLPs displayed intact full-length spike, whereas a substantial amount of the spike protein generated S1 fragments in the case of inactivated SARS-CoV-2 virions (Figure 1H). 6p-VLPs specifically bound to human ACE2 receptor coated beads but not to recombinant IL- $1 \beta$ coated control beads (Figure S1), demonstrating the specificity of the VLP-expressed spike protein towards the host receptor. VLPs retained their intact antigenic content even when incubated at $40^{\circ} \mathrm{C}$ up to 3 days (Figure S2A). Furthermore, they retained their morphology after adsorption to alum and $\mathrm{CpG}$ adjuvantation (Figure S2B). These results illustrate the feasibility of generating VLPs as a vaccine candidate, targeting the 4 structural proteins of SARS-CoV-2.

\section{Immunogenicity of SARS-CoV-2 VLP vaccine}

To assess the immunogenicity of the VLP vaccine, 4-8 weeks-old female BALB/c mice were subcutaneously immunized with 0.4 (low dose; LD) and $4 \mu \mathrm{g}$ (high dose; HD) 2p- or 6p-VLPs, separated by a 2-week interval. VLPs were either administered as such or in combination with K3-CpG (20 $\mu \mathrm{g} / \mathrm{mouse})$, alum (5 $\mu \mathrm{g} /$ mouse) or both. Mice immunized with LD or HD VLP had detectable anti-S-binding IgG and IgG1 after primary (Figure 2A) and booster injections (Figure 2B). As expected, magnitude of the secondary anti-S IgG response in all HD groups was substantially higher than their LD counterparts $(5.2,12.4,12.2$ and 8.5fold for 6p-VLP, 6p-VLP+K3-CpG, 6p-VLP+Alum, 6p-VLP+ K3-CpG +Alum groups, respectively; Figure 2B). 6p-VLP+ K3-CpG +Alum elicited 2.7-fold more anti-S IgG when compared to its formulated 2p-VLP counterpart (Figure 2B), indicating that adjuvanted hexaproline stabilized $\mathrm{S}$ is more immunogenic than the 2p-stabilized spike containing VLPs. Neither Alum, nor K3-CpG or K3-CpG /Alum combination further elevated anti-S IgG or IgG1 titers. However, compared to HD VLP alone, only the K3-CpG adjuvanted groups elicited significant anti-S IgG2a (13- and 9-fold for 6p-VLP HD+ K3-CpG and 6p-VLP HD+Alum+ K3-CpG when compared to HD 6p-VLP alone group, Figure 2B), demonstrating the preferential Th1 skewing immunostimulatory activity of K3-CpG ODN. Similarly, all VLP formulations elicited anti-RBD and anti-N IgG and IgG1 antibodies, whereas highest anti-RBD and anti-N IgG2a titers were stimulated only in mice immunized with HD VLP plus K3-CpG or CpG/Alum (Figures S3 A-D). Although in the BALB/c model IgG1 titers induced by HD 6p-VLP+Alum + K3-CpG well exceeded that of IgG2a, in hACE2 transgenic C57BL/6 mice, the same formulation elicited an anti-RBD IgG2c dominated response (Figure 4A; IgG2c:IgG1 ratio 3:1). Considering the role of IgG2a and/or IgG2c in viral clearance mechanisms ${ }^{35-38}$, K3-CpG ODN adjuvantation confers an advantage over non-adjuvanted and/or Alum adsorbed VLP administration.

Antigen-specific helper $\mathrm{T}$ cell responses were also investigated in immunized mice. Following restimulation with recombinant spike or nucleocapsid, splenocytes from mice immunized with $6 \mathrm{p}$-VLP or $6 \mathrm{p}$-VLP plus Alum, secreted significant amounts of Th2 cytokines IL-4, IL-5, IL-13 and IL-10 (Figure 2C and D, Figure S4A and B). In contrast, only the K3-CpG or CpG/Alum adjuvanted VLPs induced a Th1 biased IFN- $\gamma$ response but no Th2 associated cytokines (Figure $2 \mathrm{C}$ and D, FigS4A and B), suggesting that 6p-VLP/Alum/K3CpG vaccination would prevent Th2-biased immune responses and therefore avoid Th2-dependent vaccineassociated enhanced respiratory disease (VAERS). ${ }^{39-41}$

To study the effect of the formulation dose on VLP immunogenicity, BALB/c mice were subcutaneously immunized with 6 different doses (ranging from 24 to $0.75 \mu \mathrm{g}$ ) of 6p- VLP/Alum/K3-CpG and IgG titers against the whole inactivated virus was determined by ELISA (Figure 3A). The effective concentration at $50 \%$ (EC50), was then determined by a non-linear regression curve fit in GraphPad Prism (Figure 3A). The EC50 for the 6p- VLP/Alum/K3-CpG vaccine was determined to be $2.83 \mu \mathrm{g}$. 
To test the HD 6p-VLP+Alum+K3-CpG immunogenicity in different animal species, rats were immunized subcutaneously with $40 \mu \mathrm{g}$ of $6 \mathrm{p}-\mathrm{VLP} / \mathrm{K} 3-\mathrm{CpG} /$ Alum 2 weeks apart and live virus neutralizing antibody titers were evaluated 2 weeks after booster injection (Figure 3B). Similarly, ferrets were vaccinated either with a $10 \mu \mathrm{g}$ or a $40 \mu \mathrm{g}$ dose of the $6 \mathrm{p}-\mathrm{VLP}$ vaccine and live virus neutralizing antibody titers were determined 2 weeks after priming and booster injections (Figure 3C). 6p-VLP/Alum/K3-CpG combination induced robust neutralizing antibodies against live SARS-CoV-2 in rats and ferrets. These data indicate that $6 \mathrm{p}-$ $\mathrm{VLP} /$ Alum/K3-CpG formulation is a potent immunogen that can elicit virus-neutralizing activity in multiple species. VLPs expressing either WT 6p-S or Alpha variant 6p-S were also synthesized and then formulated with alum/CpG ODN to test their immunogenicity in C57BL/6 mice. In alpha 6p-S VLPs, spike protein expression was more enhanced compared to WT 6p-S VLPs (Figure 3 D). Consistently, alpha 6p-S VLPs elicited higher levels of anti-S and anti-inactivated virus (Wuhan) IgG in comparison to WT 6p-S VLPs, whereas anti-N IgG levels remained similar (Figure 3E). Antibodies raised against WT, Alpha, Beta and Gamma variant RBDs were also analyzed (Figure 3F). Alpha 6p-S VLPs elicited ? 37.3- 20.5- 1.7- and 11.9-fold more anti-WT, anti-alpha, anti-beta and anti-gamma RBD IgG, respectively, when compared to WT 6p-S VLP immunized mice (Figure 3F). These results suggest that alpha 6p-S expressing VLPs might be advantageous over their WT 6p-S VLP counterpart in eliciting a broader cross-protective response against variant RBDs

\section{Protective Efficacy of the SARS-CoV-2 VLP vaccine in K18-hACE2 transgenic mice}

To evaluate the immunoprotective activity of the $6 \mathrm{p}-\mathrm{VLP}$ vaccine against challenge with authentic SARS-CoV-2, K18-hACE2 transgenic mice (Jackson Laboratories, USA) were immunized with $8 \mu \mathrm{g}$ 6pVLP/Alum/K3-CpG (high dose; HD) on days zero and 14. A low dose vaccine group (2 $\mu \mathrm{g}$; low dose; LD) was also included to identify the potential of the VLP vaccine to induce VAERS when suboptimal antibodies are generated. 14 days after booster, mice immunized with the HD VLP vaccine induced significant levels of anti-RBD IgG, IgG1 and IgG2c when compared to placebo group (Figure 4A). In contrast, the $\mathrm{LD}$ vaccine generated only low titers of RBD-specific IgG2c (Figure 4A). Virus Neutralizing antibody titers (VNT) against original Wuhan or the B.1.1.7 UK variant live viruses was also measured from serum of HD 6p-VLP+Alum+K3-CpG immunized mice, 2 weeks after booster injection (Figure 4B). There was an average of 1.4-fold reduction in VNTs against the more transmissible B.1.1.7 UK variant when compared to the authentic virus, suggesting that the VLP vaccine might be effective against this specific variant of concern.

On day 21 after booster injection, mice were intranasally challenged with $10^{5}$ pfu of live SARS-CoV-2 (Wuhan strain) on 3 consecutive days. One week after the last instillation, lungs were harvested for histopathological evaluation (Figure 4D and E). Histomorphometric evaluations were based on the following criteria: (i) Inflammation was semi-quantitatively scored between 0 and 5 in the perivascular, peribronchiolar, subpleural regions and in the whole section. ${ }^{42}$ (ii) Total lung injury was evaluated based on the American Thoracic Society's acute lung injury score. ${ }^{43}$ The parenchymal inflammation area was quantitatively evaluated. ${ }^{44}$

Untreated/unchallenged healthy K18-hACE2 transgenic mouse lung samples (negative control), exhibited low grade local parenchymal inflammation at the periphery (Fig. 4D). Alveolar integrity was preserved without interalveolar septum thickening, intra-alveolar inflammatory cell infiltration or protein debris accumulation. High-dose vaccine prevented perivascular $(\mathrm{p}<0.0001)$, peribronchiolar $(\mathrm{p}=0.0002)$, subpleural $(\mathrm{p}<0.0001)$ and total $(\mathrm{p}<0.0001)$ lung parenchymal inflammation when compared to the placebo group (Figure $4 \mathrm{D}$ and E). Minimal inflammation scores equivalent to healthy animals were recorded in the high-dose vaccine group (Fig. 4E).

High-dose vaccine significantly reduced acute lung injury score consisting of inflammatory cell infiltration in the alveolar lumen and interstitial space, hyaline membrane formation, protein debris in the airways, and thickening of the interalveolar septum compared to that of the placebo group (Figure 4D). Lung specimens from animals vaccinated with high-dose VLP had low injury scores similar to healthy lung specimens (Figure $4 \mathrm{E})$. Placebo and low-dose vaccine failed to prevent acute lung injury and presented with parenchymal consolidation with diffuse infiltration of mononuclear cells and macrophages, thickened interalveolar septa to varying degrees, and hyaline membranes at the alveolar walls facing the lumen (Figure 4D). The high-dose 
vaccine group generally exhibited a limited and mild parenchymal infiltration at peribronchiolar regions.

These results indicate that $6 \mathrm{p}-\mathrm{VLP}$ vaccination confers immunoprotective activity against SARS-CoV-2 challenge and a suboptimal vaccine dose does not exacerbate virus-induced immunopathology.

\section{Discussion}

Several highly effective and safe SARS-CoV-2 vaccines have been approved and are widely administered to the populations of several countries as an indispensable measure in controlling the current pandemic. Almost all of these vaccines are based on the spike antigen and elicit neutralizing antibodies especially against the receptor-binding motif, the least conserved region of the spike antigen. With the emergence of new SARS$\mathrm{CoV}-2$ variants of concern and in light of evidence of reduced neutralization activity against some of the VOCs, vaccines that incorporate multiple antigens that are not under selective antibody pressure, could in theory contribute to long-term protective immunity through expanding the breath of virus-specific $\mathrm{T}$ cell responses.

In this respect, herein, we described the development and immunogenicity of SARS-CoV-2 VLP vaccine that incorporates the 4 structural proteins of the virus, all of which possess $\mathrm{T}$ cell epitopes. ${ }^{24-26,45-46}$

Our results showed that HEK-293 cells transfected with SARS-CoV-2 structural proteins reproducibly generated VLPs that were similar in size and physical form to the authentic virus. VLPs expressing the hexaproline stabilized prefusion spike antigen adjuvanted with Alum plus K3 CpG ODN elicited high levels of anti-S, anti-RBD, anti-N IgG and live virus neutralizing antibodies in mice, rats and ferrets. Of note, only the $\mathrm{CpG}$ ODN adjuvanted groups induced IgG2a in immunized BALB/c mice, consistent with an immune response characterized by CpG ODN-mediated Th1-type cytokines. ${ }^{47}$ Similarly, only the CpG or CpG/Alum adjuvanted VLP vaccine triggered S- and N-specific Th1- but not Th2-dominated cytokine secretion from T cells. Vaccine adjuvants are of utmost importance in enhancing and directing the adaptative immune response to protein antigens. The widely used vaccine adjuvant alum has a strong Th2 bias. CpG ODN aids in redirecting alum-induced strong Th2 responses towards the Th1 axis. ${ }^{48}$ Differently, following injection, CpG ODN adjuvants locate less efficiently to draining lymph nodes in species larger than mice. ${ }^{49}$ This drawback can be overcome through formulating the antigen and K3-CpG ODN together with alum to facilitate their delivery to lymph nodes. Our data also indicate that VLPs expressing hexaproline stabilized alpha variant spike elicited a more potent response against WT and variant RBDs compared to $6 \mathrm{p}-\mathrm{WT} \mathrm{S}$ incorporating VLPs. Whether this is due to enhanced spike expression in VLPs or a change in immunogenicity of the variant spike, remains to be determined. ${ }^{50}$

In summary, based on the immunogenicity data presented herein, the CpG ODN/alum adjuvanted 6p-VLP vaccine (VLP-58-1023-Al-K3) is currently being evaluated in a phase 1 human clinical trial (NCT04818281).

\section{AUTHOR CONTRIBUTIONS}

MICY, MG and IG conceptualized the project. MG and IG led the study. These authors acted as co-second authors: EMI, AB, NT, NSY, NG, MY, IE. ICY, designed protocols, and carried out experiments together with, BG, BS, TB, YA, IB, AGB, BI, HAS, BK, YC, TY, IA, ICA, and GA. Under the supervision of PK, SBC, ECD and MG conducted histopathological investigations and performed TEM analyses. OE, provided inactivated SARS-CoV-2 virus samples. FC, supervised VNT assays and carried out experiments together with SU, HE, CA. Challenge experiments were supervised by ST and carried out by HP and ISY. HEZ coordinated the GMP production of VLPs in Nobel Pharma. MG and IG wrote the manuscript, with input from all authors..

\section{CONFLICT OF INTEREST}

There are no financial conflict of interest to declare by the Authors.

\section{REFERENCES}


1. McGill COVID19 Vaccine Tracker Team, COVID19 Vaccine Tracker; https://covid19.trackvaccines.org/vaccines/.

2. Baden LR, El Sahly HM, Essink B, et al. Efficacy and Safety of the mRNA-1273 SARS-CoV-2 Vaccine. N Engl J Med. 2021;384(5):403-416. doi:10.1056/NEJMoa2035389

3. Polack FP, Thomas SJ, Kitchin N, et al. Safety and Efficacy of the BNT162b2 mRNA Covid-19 Vaccine. N Engl J Med. 2020;383(27):2603-2615. doi:10.1056/NEJMoa2034577

4. Voysey M, Clemens SAC, Madhi SA, et al. Safety and efficacy of the ChAdOx1 nCoV-19 vaccine (AZD1222) against SARS-CoV-2: an interim analysis of four randomised controlled trials in Brazil, South Africa, and the UK [published correction appears in Lancet. 2021 Jan 9;397(10269):98]. Lancet. 2021;397(10269):99-111. doi:10.1016/S0140-6736(20)32661-1

5. Logunov DY, Dolzhikova IV, Shcheblyakov DV, et al. Safety and efficacy of an rAd26 and rAd5 vector-based heterologous prime-boost COVID-19 vaccine: an interim analysis of a randomised controlled phase 3 trial in Russia [published correction appears in Lancet. 2021 Feb 20;397(10275):670]. Lancet. 2021;397(10275):671-681. doi:10.1016/S0140-6736(21)00234-8

6. Sadoff J, Gray G, Vandebosch A, et al. Safety and Efficacy of Single-Dose Ad26.COV2.S Vaccine against Covid-19. N Engl J Med. 2021;384(23):2187-2201. doi:10.1056/NEJMoa2101544

7. Hoffmann M, Arora P, Groß R, et al. SARS-CoV-2 variants B.1.351 and P.1 escape from neutralizing antibodies. Cell. 2021;184(9):2384-2393.e12. doi:10.1016/j.cell.2021.03.036

8. Tada T, Dcosta BM, Samanovic-Golden M, et al. Neutralization of viruses with European, South African, and United States SARS-CoV-2 variant spike proteins by convalescent sera and BNT162b2 mRNA vaccine-elicited antibodies. Preprint. bioRxiv. 2021;2021.02.05.430003. Published 2021 Feb 7. doi:10.1101/2021.02.05.430003

9. Garcia-Beltran WF, Lam EC, St Denis K, et al. Multiple SARS-CoV-2 variants escape neutralization by vaccine-induced humoral immunity [published correction appears in Cell. 2021 Apr 29;184(9):2523]. Cell. 2021;184(9):2372-2383.e9. doi:10.1016/j.cell.2021.03.013

10. Ikegame S, Siddiquey MNA, Hung CT, et al. Qualitatively distinct modes of Sputnik V vaccineneutralization escape by SARS-CoV-2 Spike variants. Preprint. medRxiv. 2021;2021.03.31.21254660. Published 2021 Apr 3. doi:10.1101/2021.03.31.21254660

11. McCallum M, Bassi J, De Marco A, et al. SARS-CoV-2 immune evasion by the B.1.427/B.1.429 variant of concern [published online ahead of print, 2021 Jul 1]. Science. 2021;eabi7994. doi:10.1126/science.abi7994

12. Cele S, Gazy I, Jackson L, et al. Escape of SARS-CoV-2 501Y.V2 from neutralization by convalescent plasma. Nature. 2021;593(7857):142-146. doi:10.1038/s41586-021-03471-w

13. Madhi SA, Baillie V, Cutland CL, et al. Efficacy of the ChAdOx1 nCoV-19 Covid-19 Vaccine against the B.1.351 Variant. N Engl J Med. 2021;384(20):1885-1898. doi:10.1056/NEJMoa2102214

14. Emary KRW, Golubchik T, Aley PK, et al. Efficacy of ChAdOx1 nCoV-19 (AZD1222) vaccine against SARS-CoV-2 variant of concern 202012/01 (B.1.1.7): an exploratory analysis of a randomised controlled trial. Lancet. 2021;397(10282):1351-1362. doi:10.1016/S0140-6736(21)00628-0

15. Zhou D, Dejnirattisai W, Supasa P, et al. Evidence of escape of SARS-CoV-2 variant B.1.351 from natural and vaccine-induced sera. Cell. 2021;184(9):2348-2361.e6. doi:10.1016/j.cell.2021.02.037

16. Kustin T, Harel N, Finkel U, et al. Evidence for increased breakthrough rates of SARS-CoV-2 variants of concern in BNT162b2-mRNA-vaccinated individuals [published online ahead of print, 2021 Jun 14]. Nat Med. 2021;10.1038/s41591-021-01413-7. doi:10.1038/s41591-021-01413-7 
17. Davis C, Logan N, Tyson G, et al. Reduced neutralisation of the Delta (B.1.617.2) SARS-CoV-2 variant of concern following vaccination. Preprint. medRxiv 2021;06.23.21259327. Published 2021 June 28. doi:10.1101/2021.06.23.21259327

18. Liu C, Ginn HM, Dejnirattisai W, et al. Reduced neutralization of SARS-CoV-2 B.1.617 by vaccine and convalescent serum [published online ahead of print, 2021 Jun 17]. Cell. 2021;doi:10.1016/j.cell.2021.06.020

19. Earle KA, Ambrosino DM, Fiore-Gartland A, et al. Evidence for antibody as a protective correlate for COVID-19 vaccines [published online ahead of print, 2021 May 24]. Vaccine. 2021;S0264-410X(21)00658-7. doi:10.1016/j.vaccine.2021.05.063

20. Peng Y, Mentzer AJ, Liu G, et al. Broad and strong memory CD4+ and CD8+ T cells induced by SARS-CoV-2 in UK convalescent individuals following COVID-19. Nat Immunol. 2020;21(11):1336-1345. doi:10.1038/s41590-020-0782-6

21. Rydyznski Moderbacher C, Ramirez SI, Dan JM, et al. Antigen-Specific Adaptive Immunity to SARSCoV-2 in Acute COVID-19 and Associations with Age and Disease Severity. Cell. 2020;183(4):996-1012.e19. doi:10.1016/j.cell.2020.09.038

22. Mathew D, Giles JR, Baxter AE, et al. Deep immune profiling of COVID-19 patients reveals distinct immunotypes with therapeutic implications. Science. 2020;369(6508):eabc8511. doi:10.1126/science.abc8511

23. Sekine T, Perez-Potti A, Rivera-Ballesteros O, et al. Robust T Cell Immunity in Convalescent Individuals with Asymptomatic or Mild COVID-19. Cell. 2020;183(1):158-168.e14. doi:10.1016/j.cell.2020.08.017

24. Grifoni A, Weiskopf D, Ramirez SI, et al. Targets of T Cell Responses to SARS-CoV-2 Coronavirus in Humans with COVID-19 Disease and Unexposed Individuals. Cell. 2020;181(7):1489-1501.e15. doi:10.1016/j.cell.2020.05.015

25. Carragher DM, Kaminski DA, Moquin A, Hartson L, Randall TD. A novel role for non-neutralizing antibodies against nucleoprotein in facilitating resistance to influenza virus. J Immunol. 2008;181(6):41684176. doi:10.4049/jimmunol.181.6.4168

26. Caddy SL, Vaysburd M, Papa G, et al. Viral nucleoprotein antibodies activate TRIM21 and induce T cell immunity. EMBO J. 2021;40(5):e106228. doi:10.15252/embj.2020106228

27. Hsieh CL, Goldsmith JA, Schaub JM, et al. Structure-based design of prefusion-stabilized SARS-CoV-2 spikes. Science. 2020;369(6510):1501-1505. doi:10.1126/science.abd0826

28. Scheiermann J, Klinman DM. Clinical evaluation of $\mathrm{CpG}$ oligonucleotides as adjuvants for vaccines targeting infectious diseases and cancer. Vaccine. 2014;32(48):6377-6389. doi:10.1016/j.vaccine.2014.06.065

29. Shirota H, Klinman DM. Recent progress concerning CpG DNA and its use as a vaccine adjuvant. Expert Rev Vaccines. 2014 Feb;13(2):299-312. doi: 10.1586/14760584.2014.863715. Epub 2013 Nov 26. PMID: 24308579; PMCID: PMC6335645.

30. Ezoe S, Palacpac NMQ, Tetsutani K, et al. First-in-human randomised trial and follow-up study of Plasmodium falciparum blood-stage malaria vaccine BK-SE36 with CpG-ODN(K3). Vaccine. 2020;38(46):72467257. doi:10.1016/j.vaccine.2020.09.056

31. Corbett KS, Edwards DK, Leist SR, et al. SARS-CoV-2 mRNA vaccine design enabled by prototype pathogen preparedness. Nature. 2020;586(7830):567-571. doi:10.1038/s41586-020-2622-0

32. Pallesen J, Wang N, Corbett KS, et al. Immunogenicity and structures of a rationally designed prefusion MERS-CoV spike antigen. Proc Natl Acad Sci U S A. 2017;114(35):E7348-E7357. doi:10.1073/pnas.1707304114

33. Wrapp D, Wang N, Corbett KS, et al. Cryo-EM structure of the 2019-nCoV spike in the prefusion conformation. Science. 2020;367(6483):1260-1263. doi:10.1126/science.abb2507 
34. Walls AC, Park YJ, Tortorici MA, Wall A, McGuire AT, Veesler D. Structure, Function, and Antigenicity of the SARS-CoV-2 Spike Glycoprotein [published correction appears in Cell. 2020 Dec 10;183(6):1735]. Cell. 2020;181(2):281-292.e6. doi:10.1016/j.cell.2020.02.058

35. Huber VC, McKeon RM, Brackin MN, et al. Distinct contributions of vaccine-induced immunoglobulin G1 (IgG1) and IgG2a antibodies to protective immunity against influenza. Clin Vaccine Immunol. 2006;13(9):981-990. doi:10.1128/CVI.00156-06

36. Huber VC, Lynch JM, Bucher DJ, Le J, Metzger DW. Fc receptor-mediated phagocytosis makes a significant contribution to clearance of influenza virus infections. J Immunol. 2001;166(12):7381-7388. doi:10.4049/jimmunol.166.12.7381

37. Mozdzanowska K, Feng J, Eid M, Zharikova D, Gerhard W. Enhancement of neutralizing activity of influenza virus-specific antibodies by serum components. Virology. 2006;352(2):418-426. doi:10.1016/j.virol.2006.05.008

38. Jayasekera JP, Moseman EA, Carroll MC. Natural antibody and complement mediate neutralization of influenza virus in the absence of prior immunity. J Virol. 2007;81(7):3487-3494. doi:10.1128/JVI.02128-06

39. Kim HW, Canchola JG, Brandt CD, et al. Respiratory syncytial virus disease in infants despite prior administration of antigenic inactivated vaccine. Am J Epidemiol. 1969;89(4):422-434. doi:10.1093/oxfordjournals.aje.a120955

40. Bolles M, Deming D, Long K, et al. A double-inactivated severe acute respiratory syndrome coronavirus vaccine provides incomplete protection in mice and induces increased eosinophilic proinflammatory pulmonary response upon challenge. J Virol. 2011;85(23):12201-12215. doi:10.1128/JVI.06048-11

41. Czub M, Weingartl H, Czub S, He R, Cao J. Evaluation of modified vaccinia virus Ankara based recombinant SARS vaccine in ferrets. Vaccine. 2005;23(17-18):2273-2279. doi:10.1016/j.vaccine.2005.01.033

42. Tian JH, Patel N, Haupt R, et al. SARS-CoV-2 spike glycoprotein vaccine candidate NVX-CoV2373 immunogenicity in baboons and protection in mice. Nat Commun. 2021;12(1):372. Published 2021 Jan 14. doi:10.1038/s41467-020-20653-8

43. Matute-Bello G, Downey G, Moore BB, et al. An official American Thoracic Society workshop report: features and measurements of experimental acute lung injury in animals. Am J Respir Cell Mol Biol. 2011;44(5):725-738. doi:10.1165/rcmb.2009-0210ST

44. Han K, Blair RV, Iwanaga N, et al. Lung Expression of Human Angiotensin-Converting Enzyme 2 Sensitizes the Mouse to SARS-CoV-2 Infection. Am J Respir Cell Mol Biol. 2021;64(1):79-88. doi:10.1165/rcmb.2020-0354OC

45. Meyers LM, Gutiérrez AH, Boyle CM, et al. Highly conserved, non-human-like, and cross-reactive SARSCoV-2 T cell epitopes for COVID-19 vaccine design and validation. NPJ Vaccines. 2021;6(1):71. Published 2021 May 13. doi:10.1038/s41541-021-00331-6

46. Matchett WE, Joag V, Stolley JM, et al. Nucleocapsid Vaccine Elicits Spike-Independent SARSCoV-2 Protective Immunity [published online ahead of print, 2021 Jun 30]. J Immunol. 2021;ji2100421. doi:10.4049/jimmunol.2100421

47. Klinman DM, Klaschik S, Sato T, Tross D. CpG oligonucleotides as adjuvants for vaccines targeting infectious diseases. Adv Drug Deliv Rev. 2009;61(3):248-255. doi:10.1016/j.addr.2008.12.012

48. Weeratna RD, Brazolot Millan CL, McCluskie MJ, Davis HL. CpG ODN can re-direct the Th bias of established Th2 immune responses in adult and young mice. FEMS Immunol Med Microbiol. 2001;32(1):6571. doi:10.1111/j.1574-695X.2001.tb00535.x

49.D5. Davis HL. Novel vaccines and adjuvant systems: the utility of animal models for predicting immunogenicity in humans. Hum Vaccin. 2008;4(3):246-250. doi:10.4161/hv.4.3.5318 
50. Lopez AM, Hecker R, Mutwiri G, van Drunen Littel-van den Hurk S, Babiuk LA, Townsend HG. Formulation with CpG ODN enhances antibody responses to an equine influenza virus vaccine. Vet Immunol Immunopathol. 2006;114(1-2):103-110. doi:10.1016/j.vetimm.2006.07.013

\section{Figure Legends}

Figure 1. Development and characterization of the VLP vaccine.(A) Schematic representation of spike, membrane, envelope and nucleocapsid protein designs. Included are a cleavable signal peptide (CD33 SP), N-terminal domain (NTD), receptor binding domain (RBD), S1/S2 boundary (S1/S2), fusion peptide (FP), heptad repeat 1 (HR1), central helix (CH), heptad repeat 2 (HR2), transmembrane domain (TM), cytoplasmic tail (CT), tobacco etch virus protease cleavage site (TEV), T4 fibritin trimerization domain (FD), thrombin cleavage site (THM) and six histidine tag sequence (His). The native polybasic furin cleavage site modifications and proline substitutions to generate the full-length WT, prefusion stabilized $2 \mathrm{p}$ and 6p spike variants are also indicated.(B) Schematic representation of VLP production, purification and formulation process. Representative transmission electron microscopy image of VLP producing HEK293 cells (C), scanning electron microscopy and atomic force microscopy images of individual VLPs ((D) and (E) are shown. (F ) TRPS size distribution measurement $(\mathrm{nm})$ of WT, $2 \mathrm{p}$ and $6 \mathrm{p}$ spike variant incorporating VLPs. Analysis of structural proteins assembled into SARS-CoV-2 VLPs by Western blot using anti-His, anti-N (G) and anti-S (H) antibodies.

Figure 2. VLPs elicit robust antibody and helper $\mathbf{T}$ cell responses in mice. BALB/c mice $(\mathrm{n}=$ 12 per group) were immunized on days 0 and 14 with 0.4 (low dose; LD) or $4 \mu \mathrm{g}$ (high dose; HD) 6p VLP or 2p VLPs without or with Alum ( $5 \mu \mathrm{g} /$ mouse), K3 CpG ODN (20 $\mu \mathrm{g} / \mathrm{mouse})$ or Alum+CpG ODN. Control $\mathrm{BALB} / \mathrm{c}$ mice were administered Alum or CpG ODN alone (black and gray). Sera were collected 2 weeks post-prime(A) and 2 weeks post-boost (B) and assessed for SARS-CoV-2 S-specific IgG, IgG1 and IgG2a by enzyme-linked immunosorbent assay (ELISA). Vaccinated groups were compared by one-way ANOVA with Dunnett's multiple comparisons test. ${ }^{*} \mathrm{P}<0.05,{ }^{* *} \mathrm{P}<0.01,{ }^{* * *} \mathrm{P}<0.001,{ }^{* * * *} \mathrm{P}<0.0001$. Data are presented as GMT \pm geometric SEM. (C) Spleens were collected 2 weeks after booster $(\mathrm{n}=6) .1 \times 10^{6} / 250 \mu \mathrm{l}$ splenocytes from naïve or immunized mice were stimulated with recombinant spike $(5 \mu \mathrm{g} / \mathrm{ml})$ in the presence of $1 \mu \mathrm{g} / \mathrm{mL}$ anti-mouse CD28. T helper cytokine levels were assessed from $48 \mathrm{~h}$ culture supernatants using the LEGENDplex MU Th Cytokine Panel (12-plex). Groups were compared by one-way ANOVA with Dunnett's multiple comparisons test. ${ }^{*} \mathrm{P}<0.05,{ }^{*} \mathrm{P}<0.01,{ }^{* * *} \mathrm{P}<0.001,{ }^{* * * *} \mathrm{P}<0.0001$. Data are presented as mean cytokine levels \pm SEM. (D)Pie charts representing the proportions of individual secreted S-specific T helper cytokines are presented.

Figure 3. Immunogenicity of the VLP vaccine in mice, rats and ferrets . (A) BALB/c mice ( $\mathrm{n}=10$ /group) were subcutaneously immunized with 6 different doses $(24-0.75 \mu \mathrm{g})$ of $6 \mathrm{p} \mathrm{VLP} /$ Alum/CpG on days 0 and 14. 2 weeks after the booster injection, IgG titers against the whole inactivated virus was determined by ELISA. ED50 was determined by non-linear regression curve fit in GraphPad Prism. (B) Sprague Dawley rats (n=5) were immunized with $40 \mu \mathrm{g}$ 6p VLP with Alum (600 $\mu \mathrm{g} / \mathrm{rat})$, K3 CpG ODN (300 $\mu \mathrm{g} / \mathrm{rat}$ ). Live virus neutralizing antibody titers were evaluated 2 weeks after booster injection. (C)Ferrets ( $\mathrm{n}=4$ /group) were vaccinated either with a $10 \mu \mathrm{g}$ or a $40 \mu \mathrm{g}$ dose of the VLP with Alum (600 $\mu \mathrm{g} / \mathrm{ferret})$ and K3 CpG ODN (300 $\mu \mathrm{g} /$ ferret). Live virus neutralizing antibody titers were determined 2 weeks after priming and booster injection. (D) Analysis of spike protein expression in WT $6 \mathrm{p}$ or alpha variant $6 \mathrm{p}-\mathrm{S}$ expressing VLPs by Western blot using anti-His (2.5 $\mu \mathrm{g}$ protein/well; 1:2 indicates two-fold diluted sample). (E-F) C57BL/6 mice ( $\mathrm{n}=5-10 /$ group) were subcutaneously immunized with $8 \mu \mathrm{g}$ of either WT $6 \mathrm{p}-\mathrm{S}$ VLP or alpha variant $6 \mathrm{p}-\mathrm{S}$ VLP vaccine on days 0 and 14 . Two-weeks after booster injection, (E) S-, inactivated virus- and $\mathrm{N}$-specific IgG titers or (F) WT, alpha, beta or gamma variant RBD-specific IgG titers were determined by ELISA. Vaccinated groups were compared by one-way ANOVA with Dunnett's multiple comparisons test. ${ }^{*} \mathrm{P}<0.05,{ }^{* *} \mathrm{P}<0.01,{ }^{* * *} \mathrm{P}<0.001,{ }^{* * * *} \mathrm{P}<0.0001$. Data are presented as GMT \pm geometric SEM.

Figure 4. Immunoprotective activity of the VLP vaccine in K18-hACE2 transgenic mice. K18hACE2 transgenic mice ( $\mathrm{n}=10$ /group) were subcutaneously immunized with $2 \mu \mathrm{g}$ (Low dose; LD) or $8 \mu \mathrm{g}$ (high dose; HD) of the VLP vaccine on days 0 and 14. 2 weeks after booster injection, (A) RBD-specific 
IgG, IgG1, IgG2c antibody titers were determined by ELISA and (B) neutralizing antibody titers against the authentic Wuhan strain and the B.1.1.7. Kent variant were determined. Groups were compared by one-way ANOVA with Dunnett's multiple comparisons test. ${ }^{*} \mathrm{P}<0.05,{ }^{* *} \mathrm{P}<0.01,{ }^{* * *} \mathrm{P}<0.001,{ }^{* * * *} \mathrm{P}$ $<0.0001$. On day 21 after booster, mice were challenged intranasally on 3 consecutive days with $50 \mu l$ of 1 $\times 10^{5} \mathrm{pfu} /$ mouse of SARS-CoV-2 (Wuhan strain). Lungs were collected 7 days after last virus instillation. (C)Infectious virus loads in lung homogenates were assessed by qRT-PCR against the nucleocapsid (NC1 and NC2). Bars represent the mean virus load ( $\mathrm{n}=10$ /group) as $1 /$ ct values. Comparisons were performed by unpaired Student's t-test; ${ }^{*} \mathrm{P}<0.05,{ }^{* *} \mathrm{P}<0.01,{ }^{* * *} \mathrm{P}<0.001,{ }^{* * * *} \mathrm{P}<0.0001$. (D)Histological micrographs showing healthy (first column), placebo (second column), low dose vaccine (third column) and high dose vaccine (fourth column) groups. A-D, Hematoxylin - Eosin (H\&E), areas marked green shows inflammed parts of the lungs; E-L, H\&E, 20x; M-P, Gomori Trichrome (GT), 40x. a, alveoli; b, bronchiole; v, blood vessel; blue arrow, protein debris; red arrow, hyaline membrane. (E)Histomorphometric measurements. The descriptive statistics were presented as median and interquartile range in all graphs except for inflamed area percent (mean +-S.D). Statistical significance $(\mathrm{p}<0.05)$ : a, compared to healthy group; b, compared to placebo group; c, compared to low dose vaccine group, d, compared to high dose vaccine group. Nonparametric variables were compared between groups using Kruskal-Wallis test. Pairwise comparisons were made with Dunn's test. Parametric variables were compared in multiple groups using one-way analysis of variance. Pairwise comparisons were performed with the Tukey test.

\section{Supporting Information}

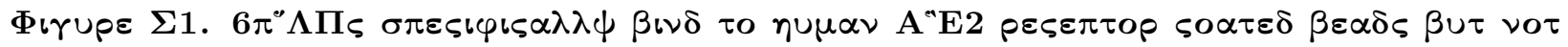

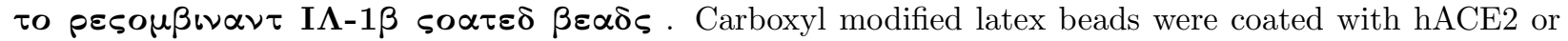
recombinant IL-1 $\beta$ and then incubated with serially diluted CFSE labeled VLPs. Beads were washed once and analyzed by flow cytometry for bead-bound VLP-CFSE signal. Top dot plots show the gating strategy and the lower plots represent bead-bound CFSE signal (y-axis) versus forward scatter (x-axis). Histograms present VLP CFSE signal intensity (mean fluorescence intensity, MFI) of serially diluted VLPs associated with negative control IL-1 $\beta$ beads (top 4 histograms) or hACE2 coated beads (lower 4 histograms).

Figure S2. Stability of SARS-CoV-2 VLPs under elevated temperature (a) and following adsorption to alum and CpG adjuvantation (b) . (a ) 6p VLPs were subjected to thermal stress for the indicated time periods. Antigen identities were assessed by western blot (anti-His Tag antibodies) and compared to a sample preserved at $5^{\mathrm{O}} \mathrm{C}$ for one week. Positions of $\mathrm{S}, \mathrm{M}$ and $\mathrm{E}$ proteins are indicated by arrows. (b) Transmission electron microscopy image of formulated 6p VLPs demonstrate that VLPs retain their morphology after adsorption to alum and $\mathrm{CpG}$ adjuvantation.

Figure S3. VLPs elicit robust antibody responses in mice.BALB/c mice ( $\mathrm{n}=12$ per group) were immunized on days 0 and 14 with 0.4 ((a ) and (b ), low dose; LD) or $4 \mu \mathrm{g}((\mathbf{c})$ and (d ) high dose; HD) 6p VLP or $2 p$ VLPs without or with Alum ( $5 \mu \mathrm{g} /$ mouse), K3 CpG ODN (20 $\mu \mathrm{g} / \mathrm{mouse})$ or Alum+CpG ODN. Control BALB/c mice were administered Alum or CpG ODN alone (black and gray). Sera were collected 2 weeks post- 2 weeks post-boost and assessed for SARS-CoV-2 RBD- (a and c ) or N-specific (b andd ) IgG, IgG1 and IgG2a by enzyme-linked immunosorbent assay (ELISA). Vaccinated groups were compared by one-way ANOVA with Dunnett's multiple comparisons test. ${ }^{*} \mathrm{P}<0.05,{ }^{* *} \mathrm{P}<0.01,{ }^{* * *} \mathrm{P}<0.001,{ }^{* * * *} \mathrm{P}$ $<0.0001$. Data are presented as GMT \pm geometric SEM.

Figure S4. 6p VLP+Alum+CpG induce S-specific TH1 dominated helper T cells. (a ) 1×106/250 $\mu \mathrm{L}$ splenocytes from naïve or immunized BALB/c mice $(\mathrm{n}=6)$ were stimulated with recombinant nucleocapsid $(20 \mu \mathrm{g} / \mathrm{ml})$ in the presence of $1 \mu \mathrm{g} / \mathrm{mL}$ anti-mouse CD28. T helper cytokine levels were assessed from $48 \mathrm{~h}$ culture supernatants using the LEGENDplex MU Th Cytokine Panel (12-plex). Groups were compared by one-way ANOVA with Dunnett's multiple comparisons test. ${ }^{*} \mathrm{P}<0.05,{ }^{* *} \mathrm{P}<0.01,{ }^{* * *} \mathrm{P}<0.001$, ${ }^{* * * *} \mathrm{P}$ $<0.0001$. Data are presented as mean cytokine levels \pm SEM. (b ) Pie charts representing the proportions of individual secreted $\mathrm{N}$-specific $\mathrm{T}$ helper cytokines are presented. 
(A)
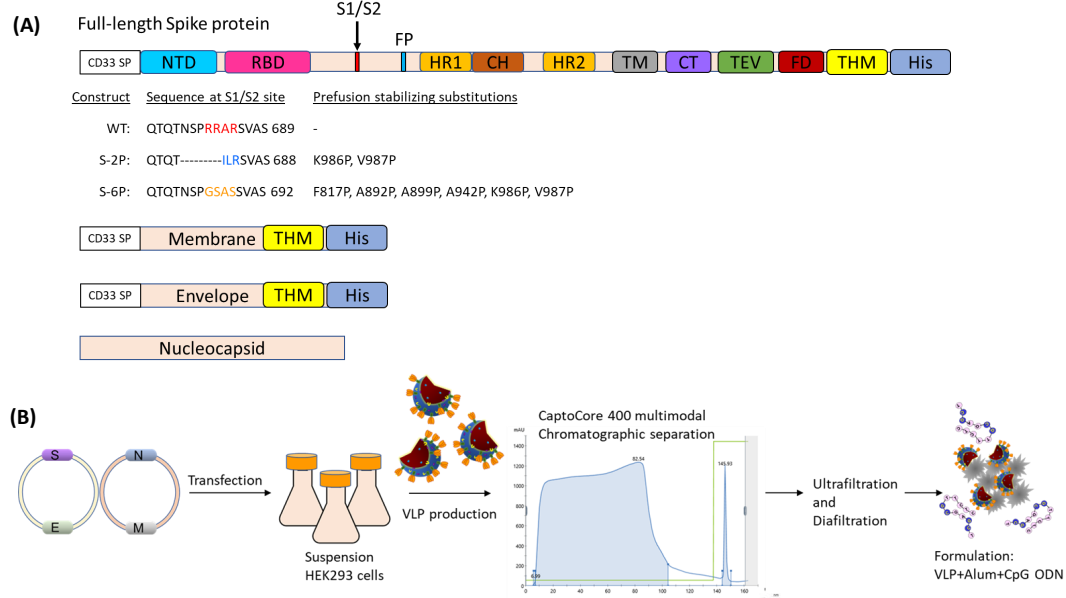

(C)

(D)
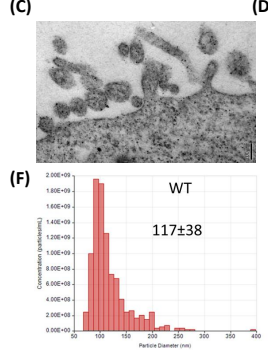

1)
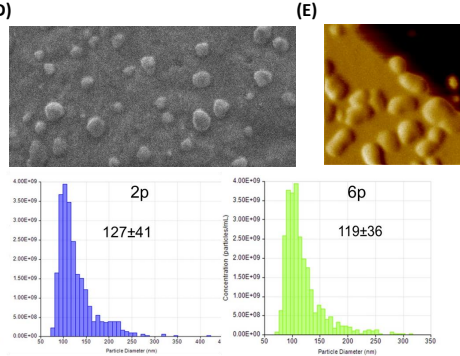

(G)

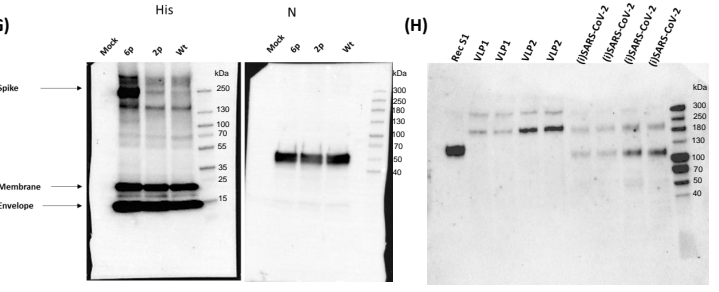



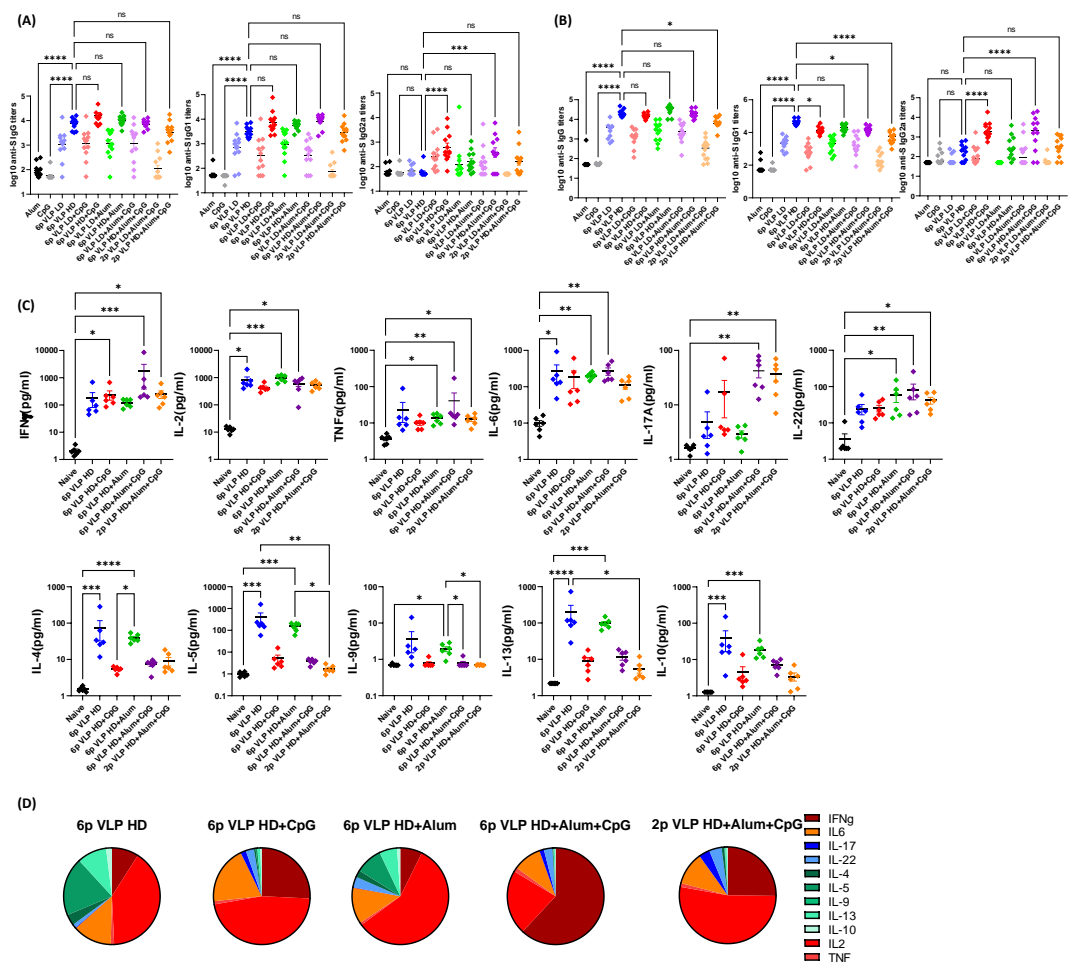
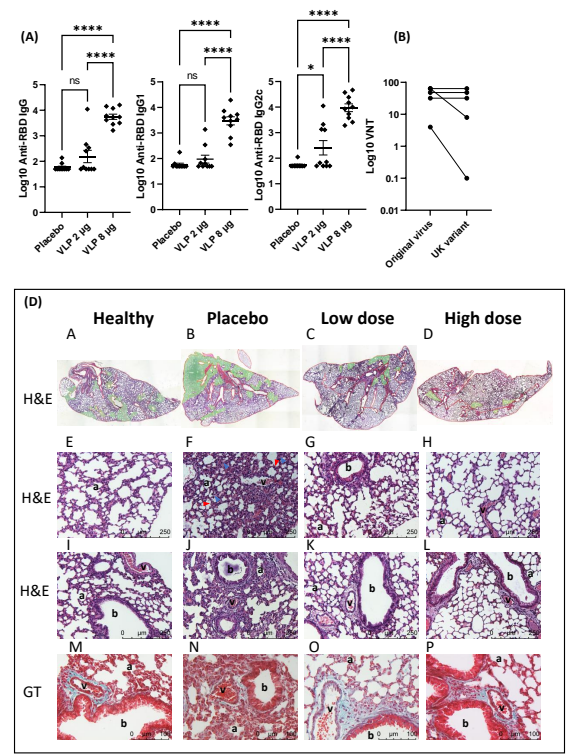

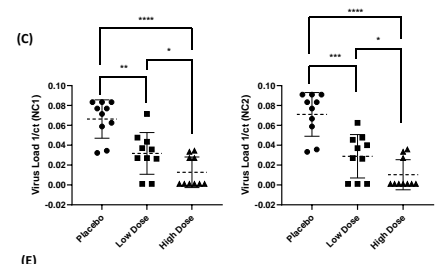

(E)

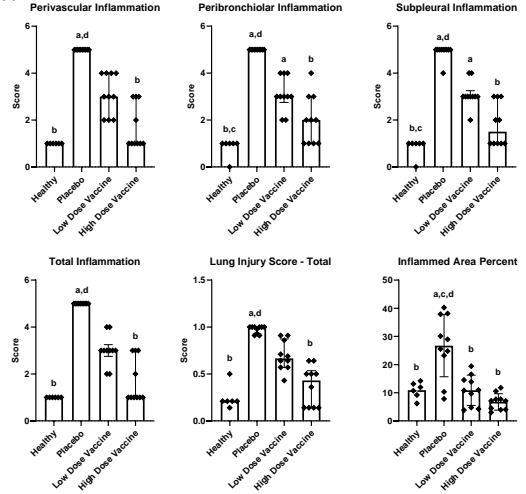

